\title{
Evaluating the Changes in Knowledge and Attitudes of Digital Library Users
}

\author{
Gemma Madle, Patty Kostkova Jane Mani-Saada and Julius R Weinberg \\ Institute of Health Sciences, City University \\ London, UK \\ g.c.madle@city.ac.uk
}

\begin{abstract}
Medical digital libraries are essentially life-critical applications providing timely access for professionals and the public to current medical knowledge and practice. This paper presents a new methodology for evaluating the impact of the knowledge within a medical digital library on users by testing their knowledge improvements and attitude changes. Using pre and post-use questionnaires we tested the impact of a small medical information website acting as an interface to the National electronic Library for Communicable Disease. The changes in user attitudes and the correlation with knowledge improvements observed indicate the potential for this methodology to be applied as a general evaluation technique of digital libraries and the impact of online information on user learning.
\end{abstract}

\section{Introduction}

Recent years have seen an explosion in the amount of health information available to patients on the Internet, but is all this newly available information making any difference to patients' behaviour? Previous usability testing of Digital Libraries (DLs) and websites has focused almost exclusively on the graphical interface issues and organization of information within the DL or website, rather than their role in changing work practices or behaviour.

As a result of this information explosion patients are becoming better informed about their healthcare decisions, armed with endless amounts of information which they may present to their clinician [1]. It is important that we can measure the effectiveness of medical DLs and health information websites in changing patient knowledge and attitudes so that the healthcare system can exploit these resources to its best advantage.

In this paper we present our research methodology for evaluating the impact of a health information website by linking the content of the site with pre-use and post-use questionnaires testing how much users knowledge of certain topics has changed by getting information from a digital library and how much this correlates to the change of attitude to the same topic.

Section 2 explains the background behind the evaluation, the DL involved and the Antimicrobial Resistance Website, which was used as a test bed for the research. In 
section 3 we describe the content structure of the Antimicrobial Resistance site and section 4 discusses the development of the pre-use and post-use questionnaires used to evaluate knowledge and attitude changes. In section 5 we present the evaluation results and also the general usability of the site. Finally, in section 6 we discuss the current status of the project, future research possibilities and in section 8 state our conclusions.

\section{Background to the Study}

This section discusses the importance of measuring the impact and usability of DLs and health information websites, how the National elelctronic Library for Communicable Disease (NeLCD) was involved in this research and the reasons for using the Antimicrobial Resistance website as a test bed.

\subsection{Usability testing of DLs}

It is becoming more and more essential to address user needs and to provide evidence that the knowledge contained within a DL has any impact on the attitudes and behaviour of the users obtaining it. Until now usability evaluations of DLs have looked mainly at the graphical interface, navigation and user acceptability $[2,3]$ rather than whether the knowledge within the DL is actually 'passed on' to the user.

For medical DLs this is of crucial importance, as they are essentially life critical applications, enabling professionals to stay up to date with current practice and empowering patients to be able to take an active role in managing their health. Recent research has shown that senior clinicians can be reluctant to allow more junior staff access to current evidence-based information, preferring instead to provide it for them or withholding it altogether [4]. This provides further support for the importance of the medical DLs that are accessible to all via the Internet and their potential role in changing the culture of the health working environment and the relationships within it.

There have been studies looking at user ability to search and find specific information on the Internet [5] but there has not been enough consideration of whether the users of DLs or health information websites have actually remembered this information, whether it has had any impact on their knowledge or attitudes. Two studies investigating the effect of a video on patient knowledge and attitudes reported increases in patient knowledge after watching the video. [6,7]. But this has yet to be applied to the web environment. Van House et al [8] agree that usability studies usually only evaluate the user interface which is not sufficient evaluation for something as complex as a digital library. They believe that the library must have an impact on the user's work.

In order to test the impact of information within DLs on users knowledge and attitudes we have developed an evaluation method based on pre and post-use questionnaires. By testing user knowledge of and attitudes towards a subject before they use a DL and then when they leave the DL it is possible to measure the immediate impact of the knowledge contained within the DL on the user. 


\subsection{Antimicrobial Resistance Website}

The NeLCD team has developed an ideal test bed for this research. A website aimed at the general public about Antimicrobial Resistance funded by the Department of Health provides an interface for the public into a subsection of a medical DL. This is a small enough collection of information to be suitable for this preliminary research and was aimed at providing information to the public to help reduce unnecessary prescribing of antibiotics i.e. changing their attitudes and eventual behaviour.

The DL used in this research is the NeLCD http://www.nelcd.co.uk, one of the Specialist Libraries of the National electronic Library for Health (NeLH http://www.nelh.nhs.uk). It provides an on-line, evidence-based, quality-tagged Internet portal to the best available evidence on prevention, treatment and investigation of communicable disease. In addition to providing a single point to available electronic resources on communicable diseases, the key value of the library is in a quality appraisal of all posted documents. This procedure is conducted in collaboration with all major professional societies and expert committees in the area of communicable disease in the UK.

The three major goals of the NeLH are [9]:

- To provide health care professionals and the public with knowledge and know-how to support health care related decisions

- To provide easy access to best current knowledge and know-how on the Internet To improve health and health care, clinical practice and patient choice.

With this in mind, in particular the first and third goals, an important consideration for the NeLCD is whether the knowledge contained within it is making any difference to the clinical practice of health professionals. It is therefore ideal to use the Antimicrobial Resistance site as a test bed for future research into the impact of the NeLCD on its users and possibly DLs in general. Although DL evaluation methods testing knowledge and attitude changes have to be designed to match the tested DL purpose and with its aim in mind, therefore, we believe that the evaluation research needs to be clearly linked to tested DL content, although there are methodological concerns which could be generalized to all types of DLs as we will show below..

\section{Structure of the Antimicrobial Resistance Website}

As discussed in section 2 the aim of the site is to contribute to reducing inappropriate prescribing of antibiotics. It will aim to do this by changing the knowledge and attitudes of the users. As the content of the website is clearly important for the later evaluation of knowledge and attitude changes we now discuss the structure of the site including the ontology and navigation aids.

\subsection{Content organization and the ontology}

To identify the scope of content for the site a search of existing 'Ask the Doctor/Expert' archives on the internet was performed to identify the most commonly asked questions about antimicrobial resistance and related issues. In addition, exist- 
ing health information sites were searched to find information available on the web. Relevant current news topics were also identified.

The different areas of content were grouped into categories using MeSH headings. These categories were then subdivided into more specific groups. In an evaluation of the NHS Direct Online website (http://www.nhsdirect.nhs.uk) Nicholas et al [10] identified four broad areas of content directly related to the main reasons that people visited the site:

- To find out more about an existing condition

- To find out what to do when they think they/or another are ill

- To help avoid illness in the first place

- To find out about NHS organizations and services

Applying these findings to the NeLCD website (as it is an NHS Direct Online 'theme month' the user group can be assumed to be very similar) and assuming that people would visit the site to either learn about antimicrobial resistance, or because they want to know how it relates to the infection that they have or to know how they can help prevent it, the original MeSH categories were organized into the following three areas:

- Learn about Antimicrobial Resistance - to include Bacteria, Viruses, Fungi, Protozoa, What Makes Us Ill, Antimicrobials, Antimicrobial Resistance, Our Immune System

- Antimicrobial Resistance and Common Illnesses - to include Colds \& Flu, Sore Throat, Sinusitis, Earache, Cystitis, Traveller's Diarrhoea, Malaria, HIV, Hospital Acquired Infection, Acne and Child Health

- Preventing Antimicrobial Resistance - to include Vaccination, Bioterrorism, Antibacterials and Food \& Farming

Each sub-category of the three areas would have a few frequently asked questions with answers that summarized and directed the reader to the relevant evidence. In addition, links to current news articles and related quality tagged resources would allow the reader to explore the topic in more depth.

The aim of the Antimicrobial Resistance site was not to reproduce information already present on the web, rather to provide an interface for the public to information held in the NeLCD.

Three types of item are present in the antimicrobial resistance site; frequently asked questions, news items and other resources. This description of documents using Dublin Core [9] will allow two methods of searching the site. A standard full-text search for those not sure exactly what they want and a more precise field search for more specific searches.

\subsection{Navigation}

The next consideration was the layout of the web pages. Navigators are important for directing a user around a site so should have an impact on the changes in knowledge and attitude. If a user cannot find knowledge within a DL how will that knowledge have any impact? 
For consistency with the NeLCD the NeLH template 'wrapper page' was used as a guide for the page layout (http://www.nelh.nhs.uk/heart/heart default.asp) along with general web design guidelines [?].

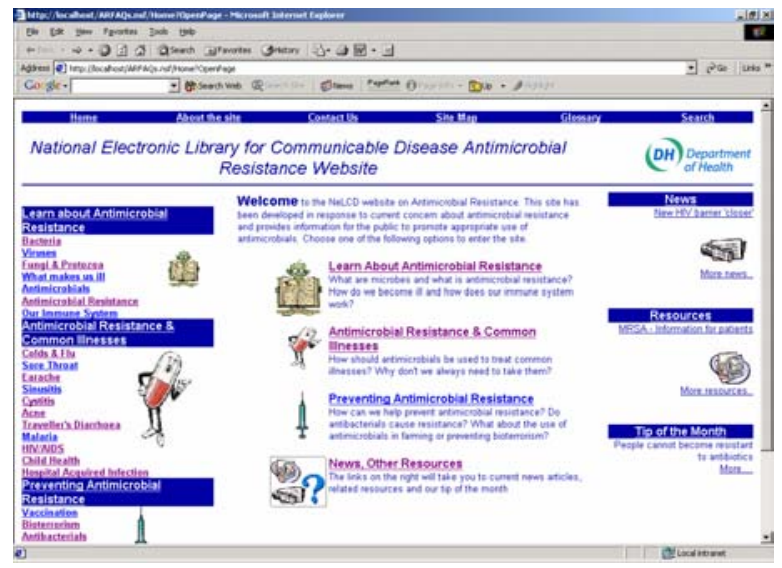

Fig. 1. Home Page of the NeLCD Antimicrobial Resistance Website

Figure 1 shows the home page of the antimicrobial resistance site. The navigation links to the frequently asked questions are situated on the left hand side of the page, structured as discussed previously in this paper. This menu is consistent throughout the site.

In the centre is the main content, this may be a frequently asked question, details of a recommended resource or news article, but in this case (the home page) a brief guide to the site, what is on offer and where to look for information. The electronic catalogue cards for the frequently asked questions, news articles and related resources are stored in the domino database and are dynamically generated as the item is requested by the user.

The right side of the page contains links to related news articles, resources and the 'tip of the month'. This content is dynamically generated from the Lotus Domino database each time the user requests a page and varies depending on the page selected by the user.

\section{User Attitude Evaluation Method Based on Pre and Post Questionnaires}

This section describes the methodology behind the development of the pre and postuse questionnaires to evaluate knowledge and attitude changes of the site with respect to the aim and content organization as discussed in section 3 .

The questionnaires both contain two identical questions (consisting of 7 true/false statements and 6 statements the user must rank their agreement with) and the postquestionnaire also a set of standard usability questions. The aims of the two identical sets of questions were: 
- Test for changes in knowledge

- Test for changes in attitudes

- Evaluate the relationship between changes in knowledge and changes in attitude

\subsection{Changes in knowledge}

To evaluate changes in knowledge the user was asked to decide whether 7 statements about antibiotics were true or false. They were then asked to rank their agreement with three statements about the use of antibiotics in a common childhood ear infection. Some of the 'answers' or correct versions of the statements were more obvious in the site than others e.g. one ('People become resistant to antibiotics' True or False?) was the subject of the current tip of the month present on every page of the site.

In contrast the following question was not explicitly answered. Users were asked to rank between 1 (strongly disagree) and 5 (strongly agree) their opinion of the following statement '10-day courses are more effective than 3-day courses in the treatment of acute otitis media (AOM)' (AOM is the common ear infection). Within a frequently asked question, evidence was provided to show that 5-day courses are as effective as 10-day courses in treating AOM but that whilst a few studies had shown similar effectiveness with 3-day courses the debate was still continuing in medical circles. Therefore the information needed to influence the user's ranking of this statement was much less explicit than other statements.

\subsection{Changes in attitude}

To evaluate changes in attitude the user was asked to rank their agreement (1 to 5 scale as before) with three statements. One of these statements was about how the user would apply information provided on the site, 'Antibiotics help to reduce the duration of pain in AOM'. A frequently asked question on the site states that 'Some children treated with antibiotics for AOM may be less likely to have pain 2-7 days after the first symptoms. But it is difficult to predict which child will benefit in this way - one study suggested that 17 must be treated for just one to benefit'. So the 'answer' is that antibiotics do reduce the duration of pain but only in one in $\mathrm{x}$ number of children. How the user applies that knowledge will be seen in their attitude to the statement after using the site i.e. to what extent they agree or disagree with it.

Two of these statements were about what the user would expect for treatment of AOM, 'Doctors should prescribe antibiotics for AOM' and 'I would expect an antibiotic for me/my child if I/they had AOM'. Answers to these statements will indicate clearly the user's attitude with respect to the use of antibiotics in AOM in general and in their own situation. The level of agreement with the statements may differ between these two situations. What a user expects for themselves or their child may be very different to what they expect for the population in general. 


\subsection{Relationship between knowledge change and attitude change}

It is all very well to improve people's knowledge but if this has no impact on their attitude or behaviour then in this case it would be a futile exercise. Therefore, the knowledge and attitude change evaluation, as discussed above, was designed in a complementary way to show the correlation between these variables. E.g., the knowledge questions on AOM were reflected in the attitude question on AOM and a question on user learning self-assessment to indicate further correlations.

To evaluate our AR test bed these results are essential as the ultimate aim of the Antimicrobial Resistance website is to contribute to reducing inappropriate prescription of antibiotics. If people know that antibiotics are not effective treatment for certain infections as a result of using the site but would still expect one from their doctor for those infections, then the site has only half done its job.

\section{Results}

In this section we describe the user sample and discuss the changes in answers and scores observed between the pre and post-use questionnaires.

\subsection{User Sample}

The antimicrobial resistance site was tested in the Science Museum, London as part of their 'live science' program. Over a period of seven days during the two February half-term weeks 227 people took part in the study. Of these 177 completed both questionnaires. This provided a rich set of data to analyze. A paired t-test was performed to test the statistical significance of changes between pairs of questionnaires. Data available on the museum visitor population indicates that a random museum visitor is more likely to have attended university or a polytechnic, more likely to have regular internet access and less likely to be unemployed than a random member of the UK public. [11] Therefore, the sample of users in this study is not strictly representative of the whole UK population.

\subsection{Changes in Knowledge}

For question one there was a significant change in the mean score ( 1 for correct answer, 0 for incorrect) of users before $($ mean $=4.33$ ) and after using the site (mean $=$ $4.90 \mathrm{p}<0.001)$. With respect to individual statements there were significant changes in the answers to four of the seven statements.

The largest change was from $9.6 \%$ of users getting the answer right to statement $1 \mathrm{~b}$ before using the site to $45.76 \%$ getting this answer correct after using the site $(\mathrm{p}<0.001)$. This reflects the visual impact of the answer to this question in the site - it was the focus of the tip of the month on the home page.

Another highly significant increase in percentage of users getting the correct answer after using the site was for statement $1 \mathrm{c}$. An increase from $57.06 \%$ to $75.14 \%$ of 
users answering correctly was observed $(\mathrm{p}<0.001)$. Again, the correct version of this statement was easily found in the first page about sore throats.

Smaller but still significant changes $(\mathrm{p}<0.05)$ were seen for statements $1 \mathrm{a}$ and $1 \mathrm{e}$, $+7.91 \%$ and $+8.47 \%$ respectively. The correct versions of both of these statements were found deeper in the content of the site, within frequently asked questions, rather than as stand-alone statements.

For the remaining three true/false statements there was a slight drop in $\%$ of users getting the correct answer although none of these changes were significant. The correct versions of these statements were not so obviously explicit within the site, relying more on the user gaining an understanding of the concept of antimicrobial resistance. For each of these statements the $\%$ of users getting the correct answer before using the site was high compared to the other four statements, ranging from $73.45 \%$ to $92.09 \%$.

The most significant change in the ranking statements (mean from 3.74 to 2.94, $\mathrm{p}<0.001)$ was for the statement about the duration of antibiotic course in the ear infection indicating that after using the site people were tending to neither disagree nor agree with the statement rather than agree. We would probably expect users to think a longer course would be more effective before they used the site and the change is in line with the information provided on the site, as discussed in section 4.1, which provides no clear answer to the statement leading users to sit on the fence rather than make a decision to agree or disagree.

\subsection{Changes in Attitudes}

With respect to changes in attitudes the statement for which there was no significant change in mean score (3.12 to 2.88) was concerned with the benefit of antibiotics in reducing pain associated with this ear infection. As the information on the site implied, there may be benefit but it is difficult to predict so we would not expect the user to be swayed one way or the other (i.e. to agree or disagree with the statement) based on this information.

There were significant decreases in the mean scores (i.e. levels of agreement) for the statements 'I would expect an antibiotic for me or my child if $\mathrm{I} /$ they had AOM' and 'Doctors should usually prescribe antibiotics for AOM' indicating that maybe this 'new' information the users had learned did have an impact on their potential behaviour.

\subsection{Relationship between changes in knowledge and changes in attitudes}

Comparing the actual changes in knowledge and attitude for individual users Figure 2 shows the relationship between users change in knowledge score and their change in attitude rank. 


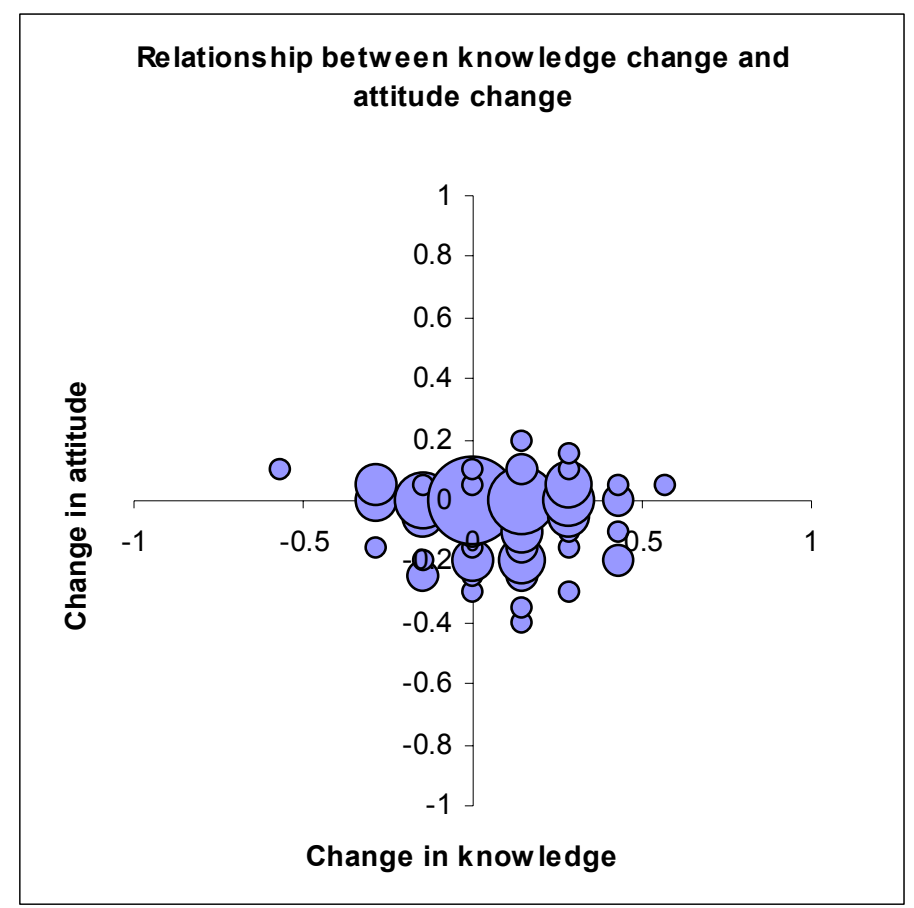

Fig. 2. Relationship between change in knowledge and change in attitude in individual users. The size of the bubbles indicates the number of users at that point

$24.24 \%$ of users increased their knowledge score and decreased their attitude rank (i.e. they were less likely to expect antibiotics for AOM). $10.10 \%$ of users did not change their knowledge score but decreased their attitude rank. As attitude change is probably more important than knowledge in effecting behavioural change we can suggest that $34.34 \%$ of users were influenced to change their attitudes in the 'direction' intended that was indicated by the aim of this site.

The mean, mode and median scores for statement $2 \mathrm{~g}$ 'I have learnt something new after using this site' were all 4 indicating that users did generally feel they had learnt from the site. This is supported by the fact that for $45.19 \%$ of users increases in knowledge scores matched the perception that they had learned from the site. In contrast $17.31 \%$ of users thought they had learned but actually decreased their knowledge score.

\subsection{General Usability Issues}

The general usability of the site was perceived by users to be good with between $70.59 \%$ and $84.97 \%$ of users agreeing or strongly agreeing with the seven usability statements. Usability scores did not vary significantly with age, gender or selfreported confidence in using the web. The mean usability score overall was 26.89 out of a maximum of $35(76.83 \%)$. 
Informal observation of users during the study identified a preference towards using the navigation menu on the left side of each page of the site rather than the search facility.

\section{Related Work}

As has been discussed previous evaluations of DLs and websites have focused more on the technical usability of the resources rather than their impact on learning and attitude changes. In particular we focus this review on medical DLs as our primary concern, however, related projects to DL evaluations are also discussed. There have been evaluations of the usability of digital libraries and health information websites. Nicholas et al [10] evaluated the NHS Direct Online site using questionnaires. The aim was to obtain feedback from users about the site so that future development could be based around their needs. The study sought to identify whether users liked the site, found it useful, what they disliked, whether they easily found information or not i.e. general self-reported usability and popularity issues.

Lancaster [12] undertook an evaluation of the use of the NeLH in five public libraries in the UK with user questionnaires and focus groups with library staff. The study found that the NeLH needs clearer navigation and more a sophisticated search facility if it is to be effectively used by public users. The aim of the NeLH is stated on their site as:

- Our aim is to provide clinicians with access to the best current know-how and knowledge to support health care related decisions. Patients, carers and the public are welcome to use this pilot, but http://www.nhsdirect.nhs.uk/NHS Direct Online (http://www.nhsdirect.nhs.uk) provides the best public gateway health information. [13]

While this aim states the focus of the NeLH on health care professionals rather than the public the report suggests that the NeLH is a potential source of more detailed information for the public than NHS Direct Online offers. However, the major barrier to effective use of the $\mathrm{NeLH}$ in the public libraries was the ability to find information at the right level. Lancaster suggests that there is some way to go before the knowledge gap between NHS Direct Online and the NeLH is bridged.

With respect to user behaviour, Sfakakis and Kapidakis [14] examined log files of the Hellenic National Documentation Centre Digital Library and found that users tended to use simple query structures for their searching and with experience reduced the number of operations they performed in a session. Eysenbach and Kohler [5] found that when searching for health information consumers were successful in trying various search terms, exploring the first few hits and subsequently refining their search strategies. They also observed that users did not often remember which websites they retrieved information from or who was behind the sites. Through interviews Van House [8] et al investigated the impact of Cypress, a digital image library on the work of its users. Following user feedback and observation of user difficulties suggestions were made for redesign of Cypress. 


\section{Project Status}

\subsection{Implementation}

The NeLCD antimicrobial resistance site was created using the Lotus Domino R5 platform. There are a selection of static html pages e.g. pages about the site, submenu pages. These static pages contain computed text that indicates latest news items or resources related to the current page. These news items and resources, along with the collection of frequently asked questions, are stored as documents in the Domino database. They are retrieved by the system on the fly as and when requested by the user. Currently only full-text search is available but Dublin Core field search will be available in the near future, and it will also be possible to cross-search across the main NeLCD site.

\subsection{Future Research}

It is planned that an online evaluation of the site, similar to this evaluation with preuse and post-use questionnaires to assess changes in knowledge and attitudes, will take place in the future. This will be supported by transaction logs to provide further information about user behaviour. In addition, more detailed mapping of the changes in answers to the site content would be useful for constructing site content e.g. how does the location or style of the answer to a question within the site affect the impact on the users' knowledge? By tracking the path of the users through the site we will be able to see if they have visited the pages that contain the answers, for how long they stayed on each page etc. It would be interesting to apply the methodology of this study to a larger study of the NeLCD. Another avenue to explore is the long-term impact on user knowledge/attitudes perhaps by a follow-up questionnaire, say three months after completion of the pre-use and post-use questionnaires.

\section{Conclusion}

This paper shows that electronic health information resources have the potential to influence users but indicates that further research is needed to determine the most effective strategies for making information available within these resources in order to have the greatest impact

We have presented a new methodology for evaluating the users knowledge and attitude change after using DLs and informational websites that is linked strongly to the content of these resources. We have tested this methodology on a small health information website and shown that it is possible to measure the impact of the knowledge contained within the site on the users knowledge and attitudes. The results show an increase in knowledge that is linked to a decrease in attitude ranking which shows that users have changed their attitude in a right way. This has implications for future evaluations of the impact of the knowledge contained within DLs on their users. 


\section{References}

1. Health on the Net Foundation "Evolution of Internet Use for health purposes", http://www.hon.ch/Survey/FebMar2001/survey.html, March 2002

2. Nielsen J., "Designing web usability: the practice of simplicity", Indianapolis, Ind., New Riders, 1999

3. Preece J., Rogers Y., Sharp H., "Interaction Design", John Wiley \& Sons Ltd, January 2002

4. Adams A., Blandford A., "Acceptability of Medical Digital Libraries", Health Informatics Journal, v8:no.2 (2002), pp58-66

5. Eysenbach G., Kohler C., "How do consumers search for and appraise health information on the world wide web? Qualitative study using focus groups, usability tests and in-depth interviews, British Medical Journal v324:no.7337 (2002) pp.573-577

6. Bauchner H., Osganian S., Smith K., Triant R., "Improving Parent Knowledge about Antibiotics: A Video Intervention", Pediatrics, v108:no.4 (2001), pp.845-850

7. Greenberg R.S., Billet C., Zahurak M., Yaster M., "Videotape increase parental knowledge about pediatric pain management", Anesthesia \& Analgesia, v89:no.4 (1999) pp.899-903

8. Van House N.A., Butler M.H., Ogle V., Schiff L., "User centered iterative design for digital libraries: the Cypress experience", D-Lib Magazine, February 1996

9. J.R. Weinberg, J. Mani-Saada, K. Smith. "The National electronic Library for Communicable Disease (NeLCD)", Poster. FIS Conference, UK, (2000)

10. Nicholas D., Huntington P., Williams P., Jordan M., "NHS Direct Online: its users and their concerns", Journal of Information Science, v28:no.4 (2002), pp305-319

11. Burch A., "Science Museum Audience Profile: A report on the first year's data", Visitor Research Group, Science Museum London, March 2002

12. Lancaster K., "Patient Empowerment", Library \& Information Update, v2:no.3 (2003), pp36-37

13. "Using NeLH" (http://www.nelh.nhs.uk/new users.asp)

14. Sfakakis M., Kapidakis S., "User Behavior Tendencies on Data Collections in a Digital Library", ECDL 2002, LCNS 2458, pp550-559 